\title{
Межзонный двухфотонный линейно-циркулярный дихроизм в полупроводниках в приближении Кейна
}

\author{
() В.Р. Расулов, Р.Я. Расулов , Б.Б. Ахмедов, И.А. Муминов \\ Ферганский государственный университет, \\ 150100 Фергана, Узбекистан \\ ๑ E-mail: r_rasulov51@mail.ru
}

Поступила в Редакцию 21 июля 2021 г.

В окончательной редакции 23 августа 2021 г.

Принята к публикации 9 сентября 2021 г.

\begin{abstract}
Классифицированы межзонные двухфотонные оптические переходы и получены выражнения для матричных элементов в узкозонном полупроводнике в зависимости от зонных параметров, степени поляризации и частоты света. Показано, что основной вклад в двухфотонный линейно-циркулярный дихроизм в узкозонных полупроводниках вносят оптические переходы, протекающие из подзоны легких дырок в зону проводимости. Проанализированы зависимости парциальных коэффициентов межзонного двухфотонного поглощения света, отличающиеся друг от друга типами оптических переходов, в зависимости от степени поляризации света, и проведен количественный анализ коэффициента линейно-циркулярного дихроизма двухфотонного поглощения света. Получены выражения для спектральной зависимости коэффициента межзонного двухфотонного поглощения света в узкозонных полупроводниках в модели Кейна.
\end{abstract}

Ключевые слова: начальное, виртуальное и конечное состояния, межзонное двухфотонное поглощения света, приближение Кейна.

DOI: 10.21883/FTP.2022.01.51813.9719

\section{1. Введение}

Первые работы по двухфотонным межзонным переходам в кристаллах были выполнены в начале 60-х годов прошлого века вскоре после появления лазеров [1-3]. При вычислении матричных элементов двухфотонных переходов в кристаллах были использованы теории возмущений по полю неполяризованной электромагнитной волны $[2,3]$, где применялась двухзонная модель Кейна.

В работах [4-7] как теоретически, так и экспериментально исследован линейно-циркулярный дихроизм (ЛЦД $)^{1}$ двух- и трехфотонного поглощения света в кристаллах кубической симметрии.

Многофотонное поглощение света в полупроводнике со сложной валентной зоной, обусловленное прямыми оптическими переходами между подзонами тяжелых и легких дырок и зависящее от степени поляризации света, было исследовано в работах [8-17]. Нелинейное межзонное однофотонное поглощение поляризованного света в полуметаллах Вейля исследовано в статье [18]. В этих работах считается, что нелинейность в зависимости коэффициента однофотонного поглощения от интенсивности света возникает за счет резонансного насыщения поглощения [19]. Это насыщение как в межзонном [18], так и во внутризонном $[9,10,16,17]$ поглощении света обусловлено фотоиндуцированным изменением функций распределения носителей тока в области импульсного пространства вблизи поверхности, определяемой законом сохранения энергии и временем релаксации, об-

\footnotetext{
${ }^{1}$ Двухфотонный линейно-циркулярный дихроизм, обусловленный межзонными оптическими переходами электронов, был предсказан Е.Л. Ивченко в работе [4]
}

ратное значение которого равно обратным значениям времен релаксаций по энергии и импульса.

В работах $[8,11,14]$ был исследован многофотонный линейно-циркулярный дихроизм (ЛЦД) в $p$-Ge в режиме развитой нелинейности, когда в поглощение вносят сопоставимый вклад $n$-фотонные процессы с $n=(1-5)$. В работах $[16,17]$ были исследованы четырехфотонные процессы в полупроводниках, обусловленные оптическими переходами между подзонами валентной зоны, с учетом эффекта когерентного насыщения.

Отметим, что в работе [7] построена теория линейноциркулярного дихроизма многофотонного межзонного поглощения различной частоты и поляризации света в полупроводниках вблизи центра зоны Бриллюэна в трехзонном приближении, когда удовлетворяется условие $\frac{2 \pi e^{2} I\left|\mathbf{e p}_{c v}\right|^{2}}{c n_{\omega} \omega^{2} m_{0}^{2}(\hbar \omega)^{2}} \ll 1$, где е и $I-$ вектор поляризации и интенсивность света, $p_{c v}=p_{c \mathbf{k}, v \mathbf{k}}=\mathbf{e p}_{c \mathbf{k}, v \mathbf{k}}-$ межзонный матричный элемент оператора импульса, $n_{\omega}-$ показатель преломления света среды на частоте $\omega, m_{0}-$ масса свободного электрона.

В настоящей работе, в отличие от работы [7], мы проводим расчеты ЛЦД межзонного двухфотонного поглощения света (ДФПС), а также спектральной зависимости коэффициента ДФПС в полупроводниках типа $\mathrm{InSb}$ в модели Кейна, где учтем вклады в многоквантовый процесс промежуточных состояний в подзонах легкой и тяжелой дырок и в отщепленной за счет спинорбитального взаимодействия валентной зоны, а также в зоне проводимости с учетом эффекта когерентного насыщения. Заметим, что в полупроводниках типа InSb энергетическое расстояние между близлежащими нижней и верхней зонами проводимости $(\tilde{\Delta})$ существенно 
больше, чем ширина запрещенной или спин-отщепленной зоны $[20,21]$, что позволяет провести дальнейшие исследования в двухзонном приближении.

\section{2. Классификация двухфотонных межзонных оптических переходов}

Известно, что вероятности одно- или многофотонных оптических переходов (ОП) и соответствующие им коэффициенты ЛЦД поглощения света определяются с использованием составных матричных элементов рассматриваемых ОП [4-17]. Поэтому в дальнейшем мы проведем анализ матричных элементов, связанных с двухфотонными ОП, характеризуемыми диаграммами Фейнмана друга выбором начальных состояний носителей тока:

a) пусть начальные состояния носителей тока расположены в подзоне тяжелых дырок $(|V, \pm 3 / 2\rangle)$ с энергией $E_{h h}$, а виртуальные состояния - в подзоне тяжелых и легких дырок $(|V, \pm 3 / 2\rangle)$ валентной зоны полупроводника (рис. $1, a, b)$. В этом случае в модели Кейна матричные элементы межзонных ОП типа $|V, \pm 3 / 2\rangle \rightarrow|m\rangle$ $\rightarrow|c, \pm 1 / 2\rangle, \quad|V, \pm 3 / 2\rangle \rightarrow|m\rangle \rightarrow|c, \mp 1 / 2\rangle \quad$ определяются матрицей в порядке $(c,+1 / 2),(c,-1 / 2)$ и $(V,+3 / 2),(V,-3 / 2)$

$$
\begin{aligned}
& \left\|M_{m^{\prime}, m}^{(2)}\right\|=\left(\frac{e A_{0}}{c \hbar}\right)^{2} P_{c V} k \\
& \times\left\|\begin{array}{cc}
\frac{2(A-B) e_{+}^{\prime} e_{z^{\prime}}}{-\hbar \omega}+\frac{e_{-}^{\prime 2} B}{E_{l h}-E_{h h}-\hbar \omega} & \frac{\sqrt{2} B e_{z}^{\prime} e_{-}^{\prime}}{E_{l h}-E_{h h}-\hbar \omega} \\
\frac{i \sqrt{2} e_{z}^{\prime} e_{+}^{\prime} B}{E_{l h}-E_{h h}-\hbar \omega} & -i\left(\frac{2(A-B) e_{-}^{\prime} e_{z^{\prime}}}{-\hbar \omega}+\frac{e_{+}^{\prime 2} B}{E_{l h}-E_{h h}-\hbar \omega}\right) \|,
\end{array}\right\|
\end{aligned}
$$

где $P_{c V}-$ параметр Кейна $[22,23], E_{l h}\left(E_{h h}\right)-$ энергия легких(тяжелых) дырок, $A, B-$ зонные параметры кристалла, $|c, \pm 1 / 2\rangle$ соответствует состояниям электронов с энергией $E_{c}$ в зоне проводимости, $k-$ волновой вектор носителей тока в конечном состоянии, $e_{ \pm}^{\prime}=e_{x}^{\prime} \pm i e_{y}^{\prime}, e_{\alpha}^{\prime}(\alpha=x, y, z)-$ проекции вектора поляризации света е на оси $x^{\prime}, y^{\prime}$, перпендикулярные волновому вектору $\mathbf{k}$. Закон сохранения энергии, определяемый для указанных ОП, выражается с помощью функции $\delta\left(E_{c}-E_{h h}-2 \hbar \omega\right), E_{h h}-$ энергия тяжелых дырок;

б) пусть начальные состояния носителей тока расположены в подзоне легких дырок валентной зоны, а виртуальные состояния - в подзонах тяжелых и легких дырок валентной зоны (рис. 2,a,b). В этом случае в модели Кейна матричные элементы межзонных ОП типа $|V, \pm 1 / 2\rangle \rightarrow|m\rangle \rightarrow|c, \pm 1 / 2\rangle$, $|V, \pm 1 / 2\rangle \rightarrow|m\rangle \rightarrow|c, \mp 1 / 2\rangle$ определяются матрицей в порядке $(c,+1 / 2),(c,-1 / 2)$ и $(V,+1 / 2),(V,-1 / 2)$ соответственно и записываются следующим образом:
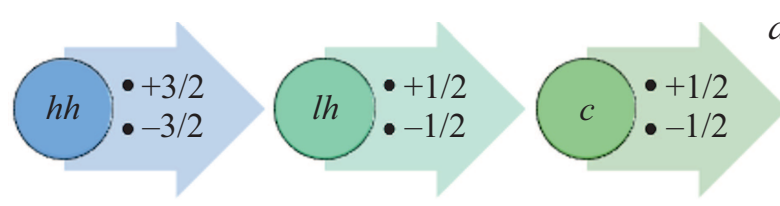

$a$
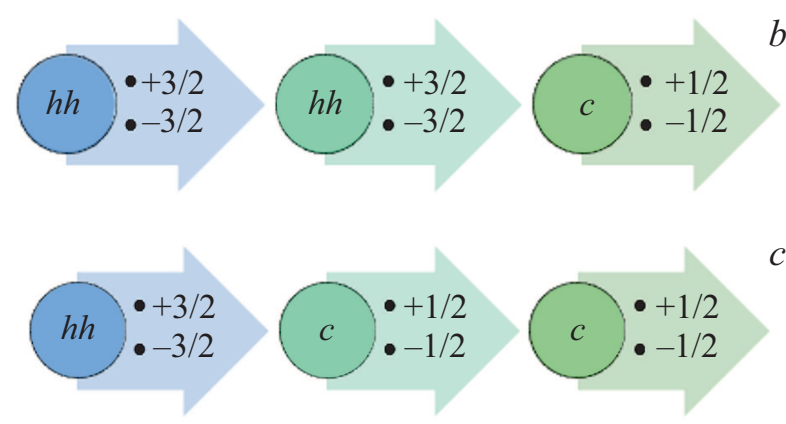

$c$
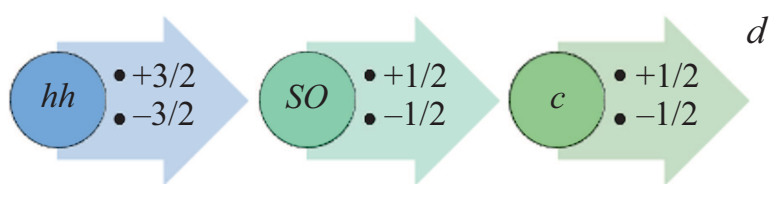

Рис. 1. Схемы двухфотонных ОП, происходящих между подзонами тяжелых дырок валентной зоны и зоны проводимости, где $h h(l h)$ - подзона тяжелых (легких) дырок, SO - зона спин-орбитального расщепления.

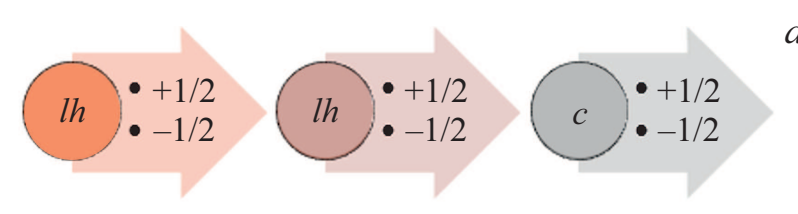

$a$

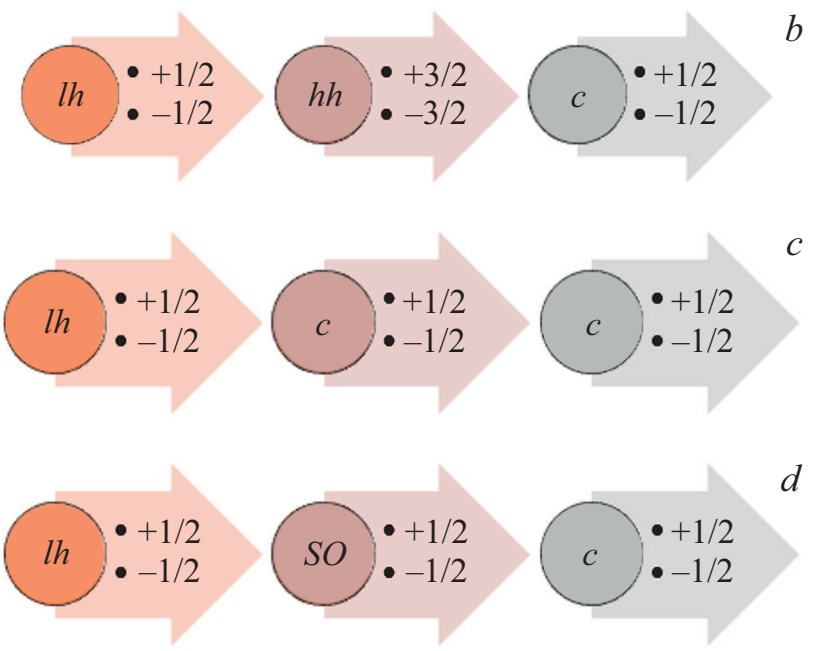

Рис. 2. Схемы двухфотонных ОП, происходящих между подзонами легких дырок валентной зоны и зоны проводимости.

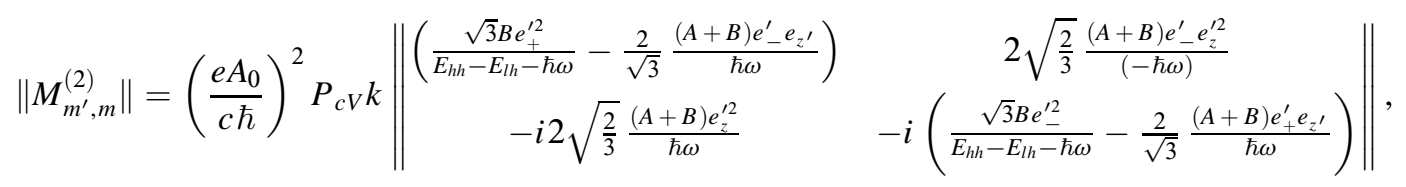


Закон сохранения энергии для этих ОП выражается с помощью функции $\delta\left(E_{c}-E_{l h}-2 \hbar \omega\right), E_{l h}-$ энергия легких дырок;

в) пусть начальные состояния носителей тока расположены в подзоне легких дырок валентной зоны, а виртуальные состояния - в зоне проводимости (рис. 2,c). В этом случае в модели Кейна матричные элементы межзонных ОП типа $|V, \pm 1 / 2\rangle \rightarrow|m\rangle \rightarrow|c, \pm 1 / 2\rangle$, $|V, \pm 1 / 2\rangle \rightarrow|m\rangle \rightarrow|c, \mp 1 / 2\rangle$ определяются матрицей в порядке $(c,+1 / 2),(c,-1 / 2)$ и $(V,+1 / 2),(V,-1 / 2)$ соответственно, записываются следующим образом:

$\left\|M_{m^{\prime}, m}^{(2)}\right\|=\frac{1}{\sqrt{3}}\left(\frac{e A_{0}}{c \hbar}\right)^{2} \frac{1}{\hbar \omega} \frac{\hbar^{2} k}{m_{c}} P_{c V} k\left\|\begin{array}{cc}e_{+}^{\prime} & \sqrt{2} e_{z}^{\prime} \\ -i \sqrt{2} e_{z}^{\prime} & i e_{-}^{\prime}\end{array}\right\|$,

где $|m\rangle-|c, \pm 1 / 2\rangle, E_{g}$ - ширина запрещенной зоны, а закон сохранения энергии для указанных ОП выражается с помощью функции $\delta\left(E_{c}-E_{l h}-2 \hbar \omega\right)$;

г) пусть начальные состояния носителей тока расположены в подзоне легких дырок валентной зоны, а виртуальные состояния - в зоне спин-орбитального расщепления (рис. $2, d$ ). В этом случае в модели Кейна матричные элементы межзонных ОП типа $|V, \pm 1 / 2\rangle \rightarrow|m\rangle$ $\rightarrow|c, \pm 1 / 2\rangle, \quad|V, \pm 1 / 2\rangle \rightarrow|m\rangle \rightarrow|c, \mp 1 / 2\rangle \quad$ определяются матрицей в порядке $(c,+1 / 2),(c,-1 / 2)$ и $(V,+1 / 2),(V,-1 / 2)$ соответственно, записываются следующим образом:
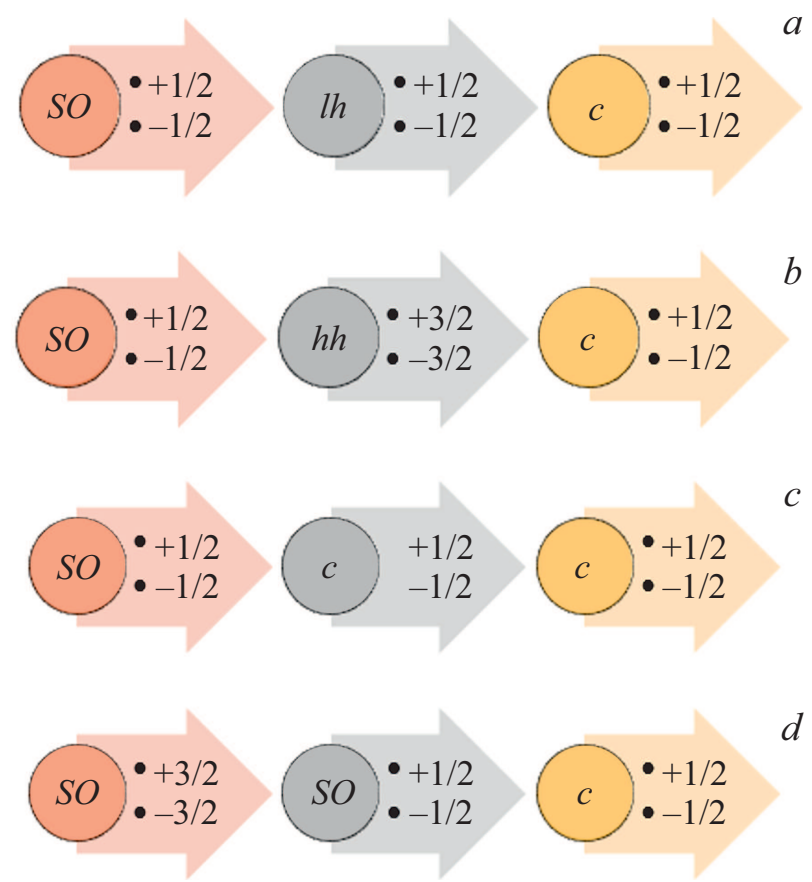

Рис. 3. Схемы двухфотонных ОП, происходящих между зоной спин-орбитального расщепления и зоной проводимости.

$$
(\widehat{M})_{c, l h}=\left(\frac{e A_{0}}{c \hbar}\right)^{2} \frac{P_{c V} B k}{2 \sqrt{3}\left(E_{h h}-E_{l h}-\hbar \omega\right)}\left[\begin{array}{cc}
6 e_{z}^{\prime} e_{-}^{\prime 2}+\left(E_{l h}+\hbar \omega\right) \frac{e_{+}^{\prime}\left(4 e_{z}^{\prime 2}+e_{\perp}^{\prime 2}\right)}{\hbar \omega} & -2 \sqrt{2}\left(E_{l h}+\hbar \omega\right) \frac{e_{z}^{\prime}\left(4 e_{z}^{\prime 2}+e_{\perp}^{\prime 2}\right)}{\hbar \omega}+i 3 e_{+}^{\prime 3} \\
3 e_{-}^{\prime 3}-i 2 \sqrt{2}\left(E_{l h}+\hbar \omega\right) \frac{e_{+}^{\prime}\left(4 e_{z}^{\prime 2}+e_{\perp}^{\prime 2}\right)}{\hbar \omega} & 6 i e_{z}^{\prime} e_{+}^{\prime 2}+i\left(E_{l h}+\hbar \omega\right) \frac{e_{+}^{\prime}\left(4 e_{z}^{\prime 2}+e_{\perp}^{\prime 2}\right)}{\hbar \omega}
\end{array}\right]
$$

где $|m\rangle=|S O, \pm 1 / 2\rangle$. Закон сохранения энергии для этого случая описывается функцией $\delta\left(E_{c}-E_{l h}-2 \hbar \omega\right)$;

д) пусть начальные состояния носителей тока расположены в подзоне тяжелых дырок валентной зоны, а виртуальные состояния - в зоне спин-орбитального расщепления (рис. 1, c). В этом случае в модели Кейна матричные элементы межзонных ОП типа $|V, \pm 3 / 2\rangle \rightarrow|m\rangle$ $\rightarrow|c, \pm 1 / 2\rangle, \quad|V, \pm 3 / 2\rangle \rightarrow|m\rangle \rightarrow|c, \mp 1 / 2\rangle \quad$ определяются матрицей в порядке $(c,+1 / 2),(c,-1 / 2)$ и $(V,+3 / 2),(V,-3 / 2)$ соответственно, записываются следующим образом:

$$
\begin{aligned}
& \left\|M_{m^{\prime}, m}^{(2)}\right\|=\frac{1}{\sqrt{6}}\left(\frac{e A_{0}}{c \hbar}\right)^{2} \frac{P_{c V}}{E_{\Delta}-E_{h h}-\hbar \omega} \\
& \times\left\|\begin{array}{cc}
-\sqrt{3} e_{+}^{\prime} H^{* \prime}-i e_{z}^{\prime}\left(G^{\prime}-F^{\prime}\right) & -i e_{-}^{\prime}\left(G^{\prime}-F^{\prime}\right)+e_{z}^{\prime} H^{* \prime} \\
i e_{z}^{\prime} H^{\prime}-e_{+}^{\prime}\left(G^{\prime}-F^{\prime}\right) & e_{z}^{\prime}\left(G^{\prime}-F^{\prime}\right)+i \sqrt{3} e^{\prime} H^{\prime}
\end{array}\right\|,
\end{aligned}
$$

где $E_{\Delta}-$ энергетический спектр носителей тока в зоне спин-орбитального расщепления, $G^{\prime}, F^{\prime}, H^{\prime}-$ первая производная по волновому вектору носителей тока от величин $G, F, H$, которые определяются формулой (24.20) из работы [22], (*) - знак комплексного сопряжения. Закон сохранения энергии выражается с помощью функции $\delta\left(E_{c}-E_{h h}-2 \hbar \omega\right)$.
Аналогичным образом можно привести выражения для оптических переходов, где начальные состояния носителей тока расположены в подзоне тяжелых дырок, а виртуальные состояния - в зоне проводимости и спинорбитального расщепления (рис. $1, c, d$ ), а также выражения для оптических переходов, где начальные состояния носителей тока расположены в зоне спин-орбитального расщепления, а виртуальные состояния - в подзоне легких и тяжелых дырок, а также в зоне проводимости и спин-орбитального расщепления (рис. 3, $a-d$ ), которые не приведены из-за краткости, но они учтены при расчетах спектральной зависимости коэффициента поглощения света и его линейно-циркулярного дихроизма.

Отметим, что волновой вектор в конечном состоянии электронов, участвующих в межзонных ОП, определяется с помощью выражения $k_{c, L}^{(2 \omega)}=\sqrt{\frac{2 \mu_{+}^{(c, L)}}{\hbar^{2}}\left(2 \hbar \omega-E_{g}\right)}$, где $m_{c}$ - эффективная масса электронов в зоне проводимости, $m_{L}$ - эффективная масса дырок в подзоне $L, L=l h(L=h h)$ - для легких (тяжелых) дырок, $\mu_{\perp}^{(c, L)}=\frac{m_{c} m_{L}}{m_{c}+m_{L}}-$ приведенная эффективная масса носи- 
телей тока. Тогда для энергий легких и тяжелых дырок справедливы следующие соотношения:

a) если ОП происходит из подзоны тяжелых дырок, тогда

$$
\begin{gathered}
E_{L=h h}\left(k_{c, L=h h}^{(2 \omega)}\right)=\frac{m_{c}}{m_{c}+m_{h h}}\left(2 \hbar \omega-E_{g}\right), \\
E_{l h}\left(k_{c, L=h h}^{(2 \omega)}\right)=\frac{m_{c} m_{h h}}{m_{l h}\left(m_{c}+m_{h h}\right)}\left(2 \hbar \omega-E_{g}\right) ;
\end{gathered}
$$

б) если ОП происходит из подзоны легких дырок, тогда

$$
\begin{gathered}
E_{L=h h}\left(k_{c, L=l h}^{(2 \omega)}\right)=\frac{m_{c} m_{l h}}{m_{h h}\left(m_{c}+m_{l h}\right)}\left(2 \hbar \omega-E_{g}\right), \\
E_{l h}\left(k_{c, L=l h}^{(2 \omega)}\right)=\frac{m_{c}}{m_{c}+m_{l h}}\left(2 \hbar \omega-E_{g}\right) .
\end{gathered}
$$

Матричные элементы двухфотонных переходов, происходящих из спин-отщепленной зоны в зону проводимости, где виртуальные состояния носителей тока расположены в подзонах валентной зоны, в зоне проводимости, а также в зоне спин-орбитального расщепления полупроводника, которые представлены на рис. 3 и определяются аналогичным образом как в вышеприведенных случаях.

Таким образом, в узкозонном кристалле были классифицированы межзонные двухфотонные ОП и получены выражнения для матричных элементов в зависимости от зонных параметров, степени поляризации и частоты света.

\section{3. Межзонное двухфотонное поглощение поляризованного света и его линейно-циркулярный дихроизм}

В этом разделе получим выражение для спектральной зависимости коэффициента межзонного ДФПС света в узкозонных полупроводниках в модели Кейна. В дальнейших расчетах используем метод расчета, предложенный в работах $[4,7,10,18])$.

Отметим, что коэффициент многофотонного поглощения света состоит из парциальных составляющих, которые по своей природе зависят от того, в какой зоне находятся носители тока как в исходном, так и в виртуальном состоянии.

В дальнейших (промежуточных) расчетах вместо $\sum_{\mathbf{k}}\left(f_{L}-f_{\text {cond }}\right) \delta\left(E_{\text {cond }}-E_{L}-2 \hbar \omega\right) F(k)$ используем выражение $\frac{1}{(2 \pi)^{3}} F\left(k_{c, L}\right) k_{c, L}^{2}$, где $k_{c, L}-$ волновой вектор, определяемый из закона сохранения энергии: $E_{c}-E_{L}-2 \hbar \omega=0$. В частности, в сферическом приближении в энергетическом спектре носителей тока, т.е. в случае $E_{L}=E_{L}^{(0)}+\frac{\hbar^{2} k^{2}}{2 m_{L}}$, волновой вектор носителей тока, участвующих в межзонных ОП, определяется как $k_{c, L}^{2}=\frac{2 \mu_{+}^{(c, L)}}{\hbar^{2}}\left(2 \hbar \omega-E_{g}\right)$, где $\mu_{+}^{(c, l h)}=\frac{m_{c} m_{L}}{m_{c}+m_{L}}-$ приведенная эффективная масса, $m_{L}$ - эффективная масса носителей тока в зоне (или подзоне) с номером $L$. В частности, $L=c$ для зоны проводимости, тогда $E_{L}^{(0)}=E_{g}$, а $L=\operatorname{lh}(h h)$ для подзоны легких (тяжелых) дырок валентной зоны, тогда $E_{L}^{(0)}=0$.

Отметим, что частотная зависимость знаменателей в матричных элементах опредеяется законом сохранения энергии, типом рассматриваемых ОП и виртуальных состояний. Например, если виртуальные состояния находятся в валентной зоне, а исходное - в подзоне тяжелых дырок, тогда знаменатель в матричном элементе данного перехода определяется выражением

$$
E_{h h}-E_{l h}-\hbar \omega=\frac{m_{c}}{m_{h h}} \frac{m_{h h}-m_{l h}}{m_{c}+m_{l h}}\left(2 \hbar \omega-E_{g}\right)+\hbar \omega,
$$

если этот переход происходит из подзоны легких дырок, тогда знаменатель в матричном элементе данного перехода определяется как

$$
E_{l h}-E_{h h}-\hbar \omega=\frac{m_{c}}{m_{l h}} \frac{m_{h h}-m_{l h}}{m_{h h}+m_{c}}\left(2 \hbar \omega-E_{g}\right),
$$

где учтены соотношения:

$$
A-B=\frac{\hbar^{2}}{2 m_{h h}}, \quad A+B=\frac{\hbar^{2}}{2 m_{l h}} .
$$

В дальнейшем рассчитаем парциальные коэффициенты двухфотонного поглощения (см., например, формулу (1) из работы [16]), отличающиеся друг от друга типом ОП, т.е. от начального, промежуточного и виртуального состояний:

a) если исходное состояние находится в подзоне тяжелых дырок валентной зоны, тогда, следуя $[16,18,19]$, коэффициент межзонного двухфотонного поглощения света можно определить выражением ${ }^{2}$

$K_{C, \pm 1 / 2 ; V, \pm 3 / 2}^{(2)}=\frac{2 \pi}{\hbar} 2 \hbar \omega \frac{1}{I} \Xi_{C, h h}^{(2)}\left(\frac{e A_{0}}{c \hbar}\right)^{2} P_{c V}^{2} k^{2}$

$\times\left\langle\frac{\left|\frac{2(A-B) e_{+}^{\prime} e_{z^{\prime}}}{-\hbar \omega}+\frac{e_{-}^{\prime 2} B}{\left(E_{l h}-E_{h h}-\hbar \omega\right)}\right|^{2}}{\sqrt{1+4 \frac{\alpha_{\omega}}{\hbar^{2} \omega^{2}}\left[\left(\frac{e A_{0}}{c \hbar}\right)^{2} P_{c V} k\right]^{2}\left|\frac{2(A-B) e_{+}^{\prime} e_{z^{\prime}}}{-\hbar \omega}+\frac{e_{-}^{\prime 2} B}{\left(E_{l h}-E_{h h}-\hbar \omega\right)}\right|^{2}}}\right\rangle$

$+\left\langle\frac{\left|\sqrt{2} B \frac{e_{z}^{\prime} e_{-}^{\prime}}{\left(E_{l h}-E_{h h}-\hbar \omega\right)}\right|^{2}}{\sqrt{1+4 \frac{\alpha_{\omega}}{\hbar^{2} \omega^{2}}\left[\left(\frac{e A_{0}}{c \hbar}\right)^{2} P_{c V} k\right]^{2}\left|\sqrt{2} B \frac{e_{z}^{\prime} e_{-}^{\prime}}{\left(E_{l h}-E_{h h}-\hbar \omega\right)}\right|^{2}}}\right\rangle$,

откуда после проведения усреднения по телесным углам волнового вектора носителей тока $(\langle\ldots\rangle)$ и без учета

\footnotetext{
2 Как здесь, так и в дальнейшем рассмотрим область малых интенсивностей света, когда применима теория возмущения.
} 
когерентного насыщения имеем

$$
\begin{aligned}
& K_{C, \pm 1 / 2 ; V, \pm 3 / 2}=\frac{8 \pi^{2}}{\hbar} \hbar \omega \frac{1}{I} \frac{\left(\mu_{+}^{c, h h}\right)^{3 / 2}}{(2 \pi)^{3} \hbar^{3}} \sqrt{2} \sqrt{2 \hbar \omega-E_{g}} f_{h h} \\
& \times\left[\frac{m_{c}}{m_{c}+m_{h h}}\left(2 \hbar \omega-E_{g}\right)\right]\left[\left(\frac{e A_{0}}{c \hbar}\right)^{2} P_{c V} k\right]^{2} \Re_{C, \pm 1 / 2 ; V, \pm 3 / 2}^{(2)}
\end{aligned}
$$

Здесь $\Xi_{C, L}^{(2)}=\sum_{\mathbf{k}}\left(f_{L}-f_{\text {cond }}\right) \delta\left(E_{\text {cond }}-E_{L}-2 \hbar \omega\right)$,

$$
\begin{aligned}
& \Re_{C, \pm 1 / 2 ; V, \pm 3 / 2}^{(2)}=\frac{1}{4 \pi} \\
& \quad \times\left\langle\left|\frac{2(A-B) e_{+}^{\prime} e_{z^{\prime}}}{(-\hbar \omega)}+\frac{e_{-}^{\prime 2} B}{\left(E_{l h}-E_{h h}-\hbar \omega\right)}\right|^{2}\right\rangle \\
& +\left\langle\left|\sqrt{2} B \frac{e_{z}^{\prime} e_{-}^{\prime}}{\left(E_{l h}-E_{h h}-\hbar \omega\right)}\right|^{2}\right\rangle \\
& =\frac{B^{2}}{15(\hbar \omega)^{2}}\left[\left(2 \frac{A-B}{B}\right)^{2} a_{C, \pm 1 / 2 ; V, \pm 3 / 2}^{(2)}\right\rangle \\
& \left.+\left(\frac{\hbar \omega}{E_{l h}-E_{h h}-\hbar \omega}\right)^{2} b_{C, \pm 1 / 2 ; V, \pm 3 / 2}^{(2)}\right],
\end{aligned}
$$

которое в сферическом приближении в энергетическом спектре носителей тока принимает вид

$$
\begin{aligned}
& \Re_{C, \pm 1 / 2 ; V, \pm 3 / 2}^{(2, s \text { fer })}=\frac{B^{2}}{15(\hbar \omega)^{2}}\left[\frac{16 m_{l h}^{2}}{\left(m_{h h}-m_{l h}\right)^{2}} a_{C, \pm 1 / 2 ; V, \pm 3 / 2}^{(2)}\right. \\
& \left.+\left(\frac{\hbar \omega\left(m_{h h}+m_{c}\right) m_{l h}}{m_{c}\left(m_{h h}-m_{l h}\right)\left(2 \hbar \omega-E_{g}\right)}\right)^{2} b_{C, \pm 1 / 2 ; V, \pm 3 / 2}^{(2)}\right],
\end{aligned}
$$

где для линейно (циркулярно)-поляризованного света $a_{C, \pm 1 / 2 ; V, \pm 3 / 2}^{(2)}=2\left(a_{C, \pm 1 / 2 ; V, \pm 3 / 2}^{(2)}=9\right), \quad a_{C, \pm 1 / 2 ; V, \pm 3 / 2}^{(2)}=3$ $\left(b_{C, \pm 1 / 2 ; V, \pm 3 / 2}^{(2)}=13\right), b_{C, \pm 1 / 2 ; V, \pm 3 / 2}^{(2)}=3\left(b_{C, \pm 1 / 2 ; V, \pm 3 / 2}^{(2)}=13\right)$. В этом случае коэффициент ЛЦД для этих ОП зависит от частоты света и зонных параметров;

б) если исходное состояние находится в подзоне легких дырок, тогда получим

$K_{C, \pm 1 / 2 ; V, \pm 3 / 2}^{(2)}=\frac{2 \pi}{\hbar} 2 \hbar \omega \frac{1}{I} \Xi_{c, l h}^{(2)}\left(\frac{e A_{0}}{c \hbar}\right)^{4} \frac{P_{c V}^{2} k^{2}}{3}$

$\times\left\langle\frac{\left|\frac{3 B e_{+}^{\prime 2}}{\left(E_{h h}-E_{l h}-\hbar \omega\right)}+2 \frac{(A+B) e_{-}^{\prime} e_{z^{\prime}}}{(-\hbar \omega)}\right|^{2}}{\sqrt{1+4 \frac{\alpha_{\omega}}{\hbar^{2} \omega^{2}}\left[\left(\frac{e A_{0}}{c \hbar}\right)^{2} \frac{P_{c V} k}{\sqrt{3}}\right]^{2}\left|\frac{3 B e_{+}^{\prime 2}}{\left(E_{h h}-E_{l h}-\hbar \omega\right)}+2 \frac{(A+B) e_{-}^{\prime} e_{z^{\prime}}}{(-\hbar \omega)}\right|^{2}}}\right\rangle$

$+\left\langle\frac{\left|2 \sqrt{2}(A+B) \frac{e_{z}^{\prime 2}}{(-\hbar \omega)}\right|^{2}}{\sqrt{1+4 \frac{\alpha_{\omega}}{\hbar^{2} \omega^{2}}\left[\left(\frac{e A_{0}}{c \hbar}\right)^{2} \frac{p k}{\sqrt{3}}\right]^{2}\left|2 \sqrt{2}(A+B) \frac{e_{z}^{\prime 2}}{(-\hbar \omega)}\right|^{2}}}\right\rangle$, или

$$
\begin{aligned}
K_{C, \pm 1 / 2 ; V, \pm 3 / 2}^{(2)}= & \frac{32 \pi}{\hbar} \hbar \omega \frac{1}{I} \Xi_{c, l h}\left(\frac{e A_{0}}{c \hbar}\right)^{2} \frac{P_{c V}^{2} k^{2}}{3} \\
& \times \Re_{C, \pm 1 / 2 ; V, \pm 1 / 2}^{(2)} .
\end{aligned}
$$

Здесь

$$
\begin{aligned}
& \Re_{C, \pm 1 / 2 ; V, \pm 1 / 2}^{(2)}=\frac{1}{4 \pi} \\
& \times\left(\left\langle\left|\frac{3 B e_{+}^{\prime 2}}{\left(E_{h h}-E_{l h}-\hbar \omega\right)}+2 \frac{(A+B) e_{-}^{\prime} e_{z^{\prime}}}{(-\hbar \omega)}\right|^{2}\right\rangle\right. \\
& \left.+\left\langle\left|2 \sqrt{2}(A+B) \frac{e_{z}^{\prime 2}}{(-\hbar \omega)}\right|^{2}\right\rangle\right)=\frac{B^{2}}{15(\hbar \omega)^{2}} \\
& \times\left[4\left(\frac{A+B}{B}\right)^{2}+\left(\frac{3 \hbar \omega}{E_{h h}-E_{l h}-\hbar \omega}\right)^{2}\right] a_{C, \pm 1 / 2 ; V, \pm 1 / 2}^{(2)}
\end{aligned}
$$

которое в сферическом приближении энергетического спектра носителей тока принимает вид

$$
\begin{aligned}
& \Re_{C, \pm 1 / 2 ; V, \pm 1 / 2}^{(2, s f e r)}=\frac{\hbar^{4}\left(m_{h h}-m_{l h}\right)^{2}}{15\left(4 \hbar \omega m_{h h} m_{l h}\right)^{2}}\left[4\left(\frac{2 m_{h h}}{m_{h h}-m_{l h}}\right)^{2}\right. \\
& \left.\quad+\left(\frac{3 \hbar \omega}{\frac{m_{c}}{m_{h h}} \frac{m_{h h}-m_{l h}}{m_{c}+m_{l h}}\left(2 \hbar \omega-E_{g}\right)+\hbar \omega}\right)^{2}\right] a_{C, \pm 1 / 2 ; V, \pm 1 / 2}^{(2)},
\end{aligned}
$$

где для линейно (циркулярно)-поляризованного света $a_{C, \pm 1 / 2 ; V, \pm 1 / 2}^{(2)}=8\left(a_{C, \pm 1 / 2 ; V, \pm 1 / 2}^{(2)}=7\right)$, коэффициент ЛЦД для этих ОП не зависит от частоты света и равен 8/7.

Пусть теперь виртуальные состояния носителей тока находятся в зоне проводимости. В этом случае:

a) если исходное состояние находится в подзоне тяжелых дырок валентной зоны, то

$$
\begin{aligned}
& K_{C, \pm 1 / 2 ; V, \pm 3 / 2}^{(2)}=\frac{2 \pi}{\hbar} 2 \hbar \omega \frac{1}{I} \Xi_{c, h h}\left(\frac{e A_{0}}{c \hbar}\right)^{4}\left(\frac{P_{c V} k}{\hbar \omega} \frac{\hbar^{2}}{m_{c}}\right)^{2} \\
& \quad \times\left\langle\frac{\left|e_{z}^{\prime} e_{-}^{\prime}\right|^{2}}{\sqrt{1+4 \frac{\alpha_{\omega}}{\hbar^{2} \omega^{2}}\left[\left(\frac{e A_{0}}{c \hbar}\right)^{2} \frac{P_{c V} k}{\hbar \omega} \frac{\hbar^{2}}{m_{c}}\right]^{2}\left|e_{z}^{\prime} e_{-}^{\prime}\right|^{2}}}\right\rangle,
\end{aligned}
$$

из которого без учета вклада эффекта когерентного насыщения в $K_{C, \pm 1 / 2 ; V, \pm 3 / 2}^{(2)}$ имеем

$$
\begin{aligned}
K_{C, \pm 1 / 2 ; V, \pm 3 / 2}^{(2)}= & \frac{2 \pi}{\hbar} 2 \hbar \omega \frac{1}{I} \Xi_{c, h h}\left(\frac{e A_{0}}{c \hbar}\right)^{4} \\
& \times\left(\frac{P_{c V} k}{\hbar \omega} \frac{\hbar^{2}}{m_{c}}\right)^{2} \frac{1}{15} a_{C, \pm 1 / 2 ; V, \pm 3 / 2}^{(2)},
\end{aligned}
$$

где для линейно (циркулярно)-поляризованного света $a_{C, \pm 1 / 2 ; V, \pm 3 / 2}^{(2)}=2\left(a_{C, \pm 1 / 2 ; V, \pm 3 / 2}^{(2)}=3\right)$, коэффициент ЛЦД для этих ОП постоянен и равен $2 / 3$; 
б) если исходное состояние находится в подзоне легких дырок валентной зоны, то

$$
\begin{aligned}
& K_{C, \pm 1 / 2 ; V, \pm 1 / 2}^{(2)}=\frac{2 \pi}{\hbar} 2 \hbar \omega \frac{1}{I} \Xi_{c, l h}\left(\frac{e A_{0}}{c \hbar}\right)^{4}\left(\frac{P_{c V} k}{\hbar \omega} \frac{\hbar^{2}}{m_{c}}\right)^{2} \\
& \times \Im_{C, \pm 1 / 2 ; V, \pm 1 / 2}^{(2)}, \\
& \Im_{C, \pm 1 / 2 ; V, \pm 1 / 2}^{(2)} \\
& =\left\langle\frac{\left|e_{z}^{\prime} e_{+}^{\prime}\right|^{2}}{\sqrt{1+4 \frac{\alpha_{\omega}}{\hbar^{2} \omega^{2}}\left[\frac{1}{\sqrt{3}}\left(\frac{e A_{0}}{c \hbar}\right)^{2} \frac{1}{\hbar \omega} \frac{\hbar^{2}}{m_{c}} P_{c V} k\right]^{2}\left|e_{z}^{\prime} e_{+}^{\prime}\right|^{2}}}\right\rangle \\
& +\left\langle\frac{\left|\sqrt{2} e_{z}^{\prime 2}\right|^{2}}{\sqrt{1+4 \frac{\alpha_{\omega}}{\hbar^{2} \omega^{2}}\left[\frac{1}{\sqrt{3}}\left(\frac{e A_{0}}{c \hbar}\right)^{2} \frac{1}{\hbar \omega} \frac{\hbar^{2}}{m_{c}} P_{c V} k\right]^{2}\left|\sqrt{2} e_{z}^{\prime 2}\right|^{2}}}\right\rangle,
\end{aligned}
$$

из которого без учета вклада эффекта когерентного насыщения в $K_{C, \pm 1 / 2 ; V, \pm 1 / 2}^{(2)}$ получим, что для света с линейной (круговой) поляризацией $\Im_{C, \pm 1 / 2 ; V, \pm 1 / 2}^{(2)}=8 / 15$ $\left(\Im_{C, \pm 1 / 2 ; V, \pm 1 / 2}^{(2)}=7 / 15\right)$, а коэффициент ЛЦД равен 7/8.

Пусть теперь виртуальные состояния носителей тока находятся в спин-орбитальной протяженной зоне. В этом случае:

a) если исходное состояние находится в подзоне тяжелых дырок валентной зоны, то получим, что

$$
\begin{aligned}
& K_{C, \pm 1 / 2 ; V, \pm 3 / 2}^{(2)}=\frac{2 \pi}{\hbar} 2 \hbar \omega \frac{1}{I} \Xi_{c, h h} \\
& \quad \times\left[\left(\frac{e A_{0}}{c \hbar}\right)^{2} \frac{1}{\sqrt{2}} \frac{B k P_{c V}}{\left(E_{\Delta}-E_{h h}-\hbar \omega\right)}\right]^{2} \Phi_{C, \pm 1 / 2 ; V, \pm 3 / 2}^{(2)} .
\end{aligned}
$$

Здесь

$$
\begin{aligned}
& \Phi_{C, \pm 1 / 2 ; V, \pm 3 / 2}^{(2)} \\
& =\left\langle\frac{\left|e_{z}^{\prime} e_{-}^{\prime}\right|^{2}}{\sqrt{1+4 \frac{\alpha_{\omega}}{\hbar^{2} \omega^{2}}\left[\left(\frac{e A_{0}}{c \hbar}\right)^{2} \frac{1}{\sqrt{2}} \frac{B k P_{c V}}{\left(E_{\Delta}-E_{h h}-\hbar \omega\right)}\right]^{2}\left|e_{z}^{\prime} e_{-}^{\prime}\right|^{2}}}\right\rangle \\
& +\left\langle\frac{\left|e_{\perp}^{\prime 2}\right|^{2}}{\sqrt{1+4 \frac{\alpha_{\omega}}{\hbar^{2} \omega^{2}}\left[\left(\frac{e A_{0}}{c \hbar}\right)^{2} \frac{1}{\sqrt{2}} \frac{B k P_{c V}}{\left(E_{\Delta}-E_{h h}-\hbar \omega\right)}\right]^{2}\left|e_{\perp}^{\prime 2}\right|^{2}}}\right\rangle,
\end{aligned}
$$

из которого без учета вклада эффекта когерентного насыщения в $K_{C, \pm 1 / 2 ; V, \pm 3 / 2}^{(2)}$ получим, что для света с линейной (круговой) поляризацией коэффициент ЛЦД равен $2 / 3$;

б) если исходное состояние находится в подзоне легких дырок валентной зоны, то коэффициент двухфотонного поглощения поляризованного света определяется как

$$
\begin{aligned}
& K_{C, \pm 1 / 2 ; V, \pm 1 / 2}^{(2)}=\frac{2 \pi}{\hbar} 2 \hbar \omega \frac{1}{I} \Xi_{c, l h} \\
& \quad \times\left[\left(\frac{e A_{0}}{c \hbar}\right)^{2} \frac{1}{\sqrt{6}} \frac{B k P_{c V}}{\left(E_{\Delta}-E_{h h}-\hbar \omega\right)}\right]^{2} \Phi_{C, \pm 1 / 2 ; V, \pm 1 / 2}^{(2)} .
\end{aligned}
$$$$
\Phi_{C, \pm 1 / 2 ; V, \pm 1 / 2}^{(2)}
$$$$
\begin{aligned}
= & \left\langle\frac{\left|3 e_{ \pm}^{\prime 2}+4 e_{z^{\prime}}^{2}\right|^{2}}{\sqrt{1+4 \frac{\alpha_{\omega}}{\hbar^{2} \omega^{2}}\left[\left(\frac{e A_{0}}{c \hbar}\right)^{2} \frac{1}{\sqrt{6}} \frac{B k P_{c V}}{\left(E_{\Delta}-E_{h h}-\hbar \omega\right)}\right]^{2}\left|3 e_{ \pm}^{\prime 2}+4 e_{z^{\prime}}^{2}\right|^{2}}}\right\rangle \\
& +\left\langle\frac{\left|e_{z}^{\prime} e_{+}^{\prime}\right|^{2}}{\sqrt{1+4 \frac{\alpha_{\omega}}{\hbar^{2} \omega^{2}}\left[\frac{1}{\sqrt{3}}\left(\frac{e A_{0}}{c \hbar}\right)^{2} \frac{1}{\hbar \omega} \frac{\hbar^{2}}{m_{c}} P_{c V} k\right]^{2}\left|e_{z}^{\prime} e_{+}^{\prime}\right|^{2}}}\right\rangle,
\end{aligned}
$$

из которого без учета вклада эффекта когерентного насыщения в $K_{C, \pm 1 / 2 ; V, \pm 3 / 2}^{(2)}$ получим, что коэффициент ЛЦД равен $3 / 2$.

Отметим, что суммарный коэффициент ДФПС $\left(K_{c, V}^{(2)}\right)$ определяется суммой вышеперечисленных парциальных коэффициентов ДФПС. Таким образом, основной вклад в ЛЦД ДФПС вносят ОП, протекающие из подзоны легких дырок в зону проводимости.

Далее вычислим спектральную зависимость суммарного коэффициента ДФПС в модели Кейна и будем использовать следующие выражения для энергетических спектров носителей тока в параболическом приближении:

$$
\begin{gathered}
E_{c}(\mathbf{k})=E_{g}+\frac{\hbar^{2} k^{2}}{2 m_{0}}+\frac{k^{2} P_{c V}^{2}\left(E_{g}+\frac{2}{3} \Delta\right)}{E_{g}\left(E_{g}+\Delta\right)}, \\
E_{h h}(\mathbf{k})=\frac{\hbar^{2} k^{2}}{2 m_{0}}, \quad E_{l h}(\mathbf{k})=\frac{\hbar^{2} k^{2}}{2 m_{0}}-\frac{2 k^{2} P_{c V}^{2}}{3 E_{g}}, \\
E_{S O}(\mathbf{k})=-\Delta+\frac{\hbar^{2} k^{2}}{2 m_{0}}-\frac{k^{2} P_{c V}^{2}}{3\left(\Delta+E_{g}\right)},
\end{gathered}
$$

где $E_{g}(\Delta)$ - ширина запрещенной (спин-орбитальной) зоны, $P_{c V}-$ параметр Кейна [23]. Тогда спектральная зависимость коэффициента двухфотонного поглощения линейно поляризованного света в области малых значений волнового вектора носителей тока имеет вид

$$
K_{c, V}^{(2)}(\omega)=K_{c, V}^{(0)} \Im_{c, V}^{(2, l)}\left(\frac{2 \hbar \omega}{E_{g}}\right),
$$

где $\quad K_{c, V}^{(0)}=\frac{4 \pi e^{2} P_{c V}}{\hbar c^{2} n_{\omega}^{2} E_{g}^{3}}, \quad E_{g} \ll E_{S O} \quad$ для $\quad$ случая $\quad l=1$, $E_{g} \gg E_{S O}$ для случая $l=2$,

$$
\begin{aligned}
\Im_{c, V}^{(2,1)}(\xi)= & \frac{4 \xi^{3 / 2}}{15 \sqrt{6}(\xi+1)^{3}}\left[480 \frac{(\xi+1)^{1 / 2}}{(3 \xi+1)^{2}}+\frac{(\xi+2)^{3 / 2}}{(\xi+1)^{5}}\right. \\
& \left.\times\left(9(\xi+1)^{4}+40(\xi+1)^{2}+96\right)\right],
\end{aligned}
$$




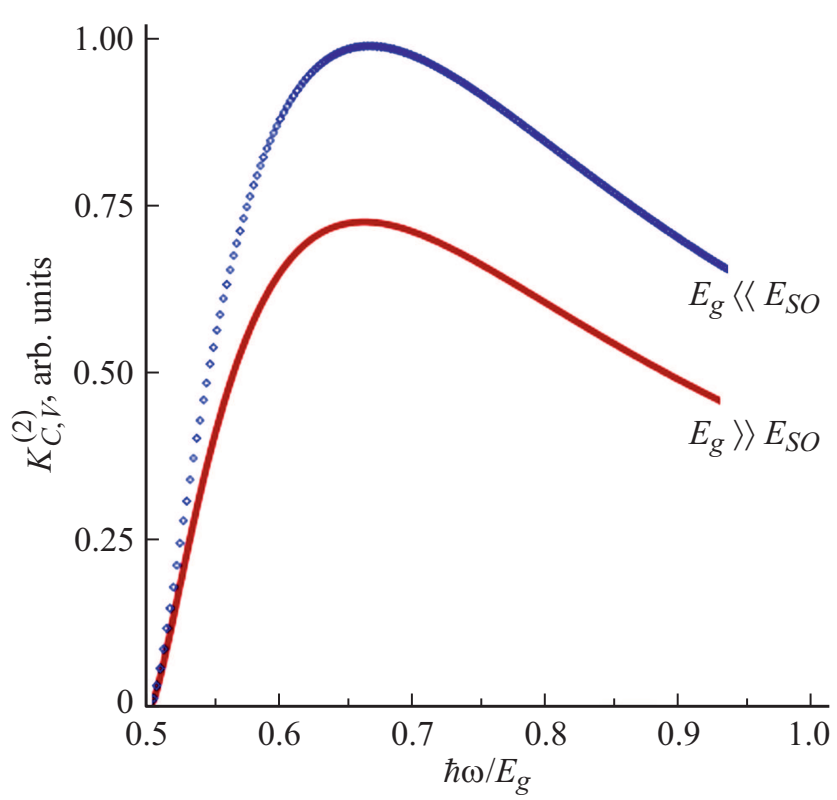

Рис. 4. Спектральная зависмость коэффициента межзонного двухфотонного поглощения линейно поляризованного света в кристалле InSb, соответствующая случаям $E_{g} \gg E_{S O}$ и $E_{g} \ll E_{S O}$.

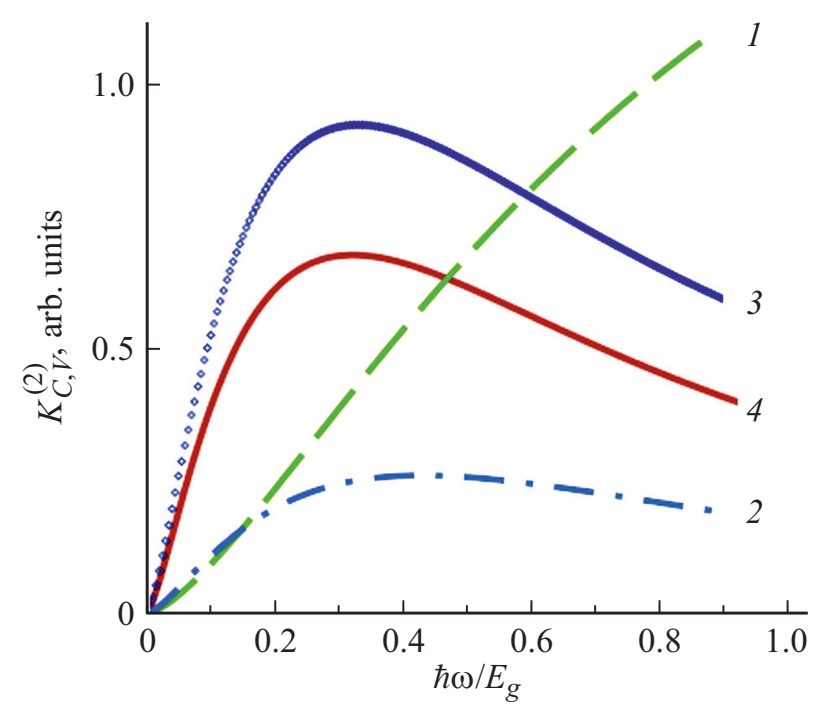

Рис. 5. Спектральная зависимость коэффициента межзонного двухфотонного поглощения линейно поляризованного света в кристалле InSb, соответствующая случаям $E_{g} \ll E_{S O}$ (кривые 1 и 3$)$ и $E_{g} \gg E_{S O}$ (кривые 2 и 4 ). Кривые 1 и 2 соответствуют параболическому, а кривые 3 и 4 непараболическому приближению в энергетическом спектре носителей тока.

$$
\begin{aligned}
\Im_{c, V}^{(2,2)}(\xi)= & \frac{32 \xi^{3 / 2}}{15(\xi+1)^{3}}\left\{36 \frac{(\xi+1)^{1 / 2}}{(3 \xi+1)^{2}}+\frac{(\xi+2)^{3 / 2}}{(\xi+1)^{5}}\right. \\
& \left.\times\left((\xi+1)^{4}+2(\xi+1)^{2}+6\right)\right\}, \\
\xi=(2 \cdot \hbar \omega- & \left.E_{g}\right) / E_{g} .
\end{aligned}
$$

На рис. 4 и 5 представлена зависимость $K_{c, V}^{(2)}(\omega)$ для InSb в двух случаях: $E_{g} \ll E_{S O}$ и $E_{g} \gg E_{S O}$. В расчетах предполагалось, что начальные состояния носителей тока полностью заняты, а конечные состояния - полностью пустые. Эти результаты показывают, что при освещении InSb линейно поляризованным светом, как в случае $E_{g} \ll E_{S O}$, так и при $E_{g} \gg E_{S O}$, спектральная зависимость $K_{c, V}^{(2)}(\omega)$ с ростом частоты увеличивается, достигает максимума, а затем уменьшается (рис. 4 и 5, кривые 3,4$)$. Это объясняется сложностью зонной структуры полупроводника в модели Кейна, которая отражается в матричных элементах и в энергетических спектрах. За счет этого возникают сложные зависимости плотности состояний и энергий как конечных, так и начальных состояний фотовозбужденных носителей тока от частоты света. Если ограничимся сферическим приближением в энергетическом спектре, тогда $K_{c, V}^{(2)}(\omega)$ будет увеличиваться с ростом частоты при условии $E_{g} \ll E_{S O}$ (рис. 4).

Отметим, что при учете анизотропии энергетического спектра электронов в валентной зоне в двухзонном приближении и в области малых значений волнового вектора носителей тока наши результаты по поляризационной зависимости коэффициента двухфотонного поглощения света в узкозонных полупроводниках совпадают с результатами работы [7]. Количественные значения зонных параметров были взяты из работы [24].

\section{4. Заключение}

В заключение сформулируем кратко основные результаты и выводы из проделанной работы.

1. Классифицированы матричные элементы межзонных двухфотонных ОП в узкозонном полупроводнике в зависимости от компонент вектора поляризации света.

2. В приближении Кейна как с учетом, так и без учета эффекта когерентного насыщения рассчитаны поляризационные и спектральные зависимости парциальных коэффициентов ДФПС и их ЛЦД, отличающихся друг от друга типом ОП.

3. На основе золотого правила квантовой механики показано, что при освещении InSb линейно поляризованным светом, как в случае $E_{g} \ll E_{S O}$, так и при $E_{g} \gg E_{S O}$, спектральная зависимость $K_{c, V}^{(2)}(\omega)$ с ростом частоты увеличивается, достигает максимума, а затем уменьшается, и этот случай объясняется сложностью зависимостей плотности состояний и энергий как конечных, так и начальных состояний фотовозбужденных носителей тока от частоты света, которые связаны с особенностями зонной структуры полупроводника в модели Кейна. Если ограничимся сферическим приближением в энергетическом спектре, тогда $K_{c, V}^{(2)}(\omega)$ будет увеличиваться с ростом частоты при условии $E_{g} \ll E_{S O}$ (рис. 5, кривая 1 ).

4. Развита теория ЛЦД, связанного межзонными двухфотонными ОП в узкозонных полупроводниках в приближении Кейна. 


\section{Конфликт интересов}

Авторы заявляют, что у них нет конфликта интересов.

\section{Список литературы}

[1] R. Loudon. Proc. Phys. Soc., 80 (4), 952 (1962).

[2] R. Braunstein. Phys. Rev., 125 (2), 475 (1962).

[3] R. Braunstein, N. Ockman. Phys. Rev., 134, 499 (1964).

[4] Е.Л. Ивченко. ФТТ, 14 (12), 3489 (1972).

[5] Е.Л. Ивченко, Е.Ю. Перлин. ФТТ, 15 (9), 2781 (1973).

[6] Е.В. Берегулин, Д.П. Дворников, Е.Л. Ивченко, И.Д. Ярошецкий. ФТП, 9 (5), 876 (1975).

[7] С.Б. Арифжанов, Е.Л. Ивченко. ФТТ, 17, 81 (1975).

[8] С.Д. Ганичев, Е.І. Ивченко, С.А. Емельянов, Е.Ю. Перлин, Я.В. Терентьев, А.В. Федоров, И.Д. Ярошецкий. ЖЭТФ, 91 (11), 1233 (1986).

[9] С.Д. Ганичев, Е.Л. Ивченко, Р.Я. Расулов, И.Д. Ярошецкий, Б.Я. Авербух. ФТТ, 35 (1), 198 (1993).

[10] Р.Я. Расулов. Дис. докт. физ.-мат. наук (СПб., ФТИ им. А.Ф. Иоффе РАН, 1993).

[11] С.Д. Ганичев, С.А. Емельянов, Е.Л. Ивченко, Е.Ю. Перлин, Я.В. Терентьев, А.В. Федоров, И.Д. Ярошецкий. ЖЭТФ, 91, 729 (1986).

[12] Р.Я. Расулов, Г.Х. Хошимов, Х. Холитдинов. ФТП, 30 (2), 274 (1996).

[13] Р.Я. Расулов. ФТП, 22 (11), 2077 (1988).

[14] Р.Я. Расулов. ФТТ, 35 (6), 1674 (1993).

[15] В.Р. Расулов, Р.Я. Расулов, Р.Р. Султонов, Б.Б. Ахмедов. ФТП, 54 (11), 1181 (2020).

[16] V.R. Rasulov, R.Ya. Rasulov, I. Eshboltaev. Phys. Solid State, 59 (3), 463 (2017).

[17] V.R. Rasulov, R.Ya. Rasulov, I. Eshboltaev. Russ. Phys. J., $58(12), 1681$ (2015).

[18] N.V. Leppenen, E.L. Ivchenko, L.E. Golub. Phys. Status Solidi B, 256, 1900305 (2019). https://doi.org/10.1002/pssb.201900305/

[19] Д.А. Паршин, А.Р. Шабаев. ЖЭТФ, 92 (4), 1471 (1987).

[20] J.-Y. You, B. Gu, S. Maekawa, G. Su. Phys. Rev. B, 102, 094432 (2020). https://doi.org/10.1103/PhysRevB.102.094432

[21] R. Mohammad, Şenay Katircioǵlu, Musa El-Hasan. J. Mater. Sci., 43, 2935 (2008). DOI 10.1007/s10853-007-1794-4

[22] Г.Л. Бир, Г.Е. Пикус. Симметрия и дебормационные эффекты в полупроводниках (М., Медиа, 2012).

[23] Е.Л. Ивченко, Р.Я. Расулов. Симметрия и реальная зонная структура полупроводников (Ташкент, Фан, 1989).

[24] I. Vurgaftman, J.R.M. Meyer, J.R. Ram-Moha. J. Appl. Phys., 89, 5815 (2001).

Редактор А.Н. Смирнов

\section{Interband two-photon linear-circular dichroism in semiconductors in the Kane approximation}

\author{
V.R. Rasulov, R.Ya. Rasulov, B.B. Akhmedov, \\ I.A. Muminov \\ Fergana State University, \\ 150100 Ferghana, Uzbekistan
}

Abstract Interband two-photon optical transitions are classified and expressions are obtained for the matrix elements in a narrow-gap semiconductor depending on the band parameters, degree of polarization, and light frequency. It is shown that the main contribution to two-photon linear-circular dichroism in narrow-gap semiconductors is made by optical transitions proceeding from the subband of light holes to the conduction band. The dependences of the partial coefficients of interband two-photon absorption of light, which differ from each other by the types of optical transitions, are analyzed depending on the degree of polarization of the light, and a quantitative analysis of the coefficient of linear-circular dichroism of two-photon absorption of light is carried out. Expressions are obtained for the spectral dependence of the coefficient of interband two-photon absorption of light in narrow-gap semiconductors in the Kane model. 